\title{
Weill Cornell Medicine Wellness Qlinic: Adapting the Student-Run Clinic Model to Expand Mental Health Services and Medical Education
}

\author{
Constance Zhou ${ }^{1}$ [ $\cdot$ Kate Fruitman ${ }^{2} \cdot$ Sarah Szwed $^{1} \cdot$ Matthew Wickersham $^{1} \cdot$ Jessica Spellun $^{3} \cdot$ Jess Zonana $^{3}$
}

Received: 29 October 2021 / Accepted: 10 January 2022 / Published online: 27 January 2022

(c) The Author(s), under exclusive licence to Springer Science+Business Media, LLC, part of Springer Nature 2022

\begin{abstract}
The Weill Cornell Medicine Wellness Qlinic (Wellness Qlinic) is a student-run mental health clinic serving the lesbian, gay, bisexual, transgender, and queer (LGBTQ+) community in New York City. Student-run clinics have successfully provided primary care to underserved communities experiencing barriers to accessing health care. Psychiatric evaluation and medication management have also been implemented in several student-run clinics, but providing sustainable psychotherapy services has been a challenge. In this paper, we present a student-run mental health program incorporating interdisciplinary trainees to provide robust short-term psychiatric treatment, including individual psychotherapy, medication management, and group therapy. Results of a chart-review study to evaluate patient engagement and treatment outcomes are presented. The Wellness Qlinic's treatment model resulted in $90 \%$ patient retention and positive clinical outcomes for patients while addressing an education and training gap in LGBTQ + mental health for multidisciplinary mental health care providers.
\end{abstract}

Keywords Student-run clinics $\cdot$ LGBTQ $+\cdot$ Healthcare disparities $\cdot$ Mental health

\section{Introduction}

Student-run clinics (SRCs) are becoming more common in U.S. medical schools. They facilitate medical education through volunteerism, while providing low-cost healthcare services to historically underserved populations (Simpson \& Long, 2007). SRCs improve student attitudes towards interprofessional healthcare teams and care for underserved patients (Shrader et al., 2010; Smith et al., 2012). SRCs predominantly target the primary care needs of uninsured patients, with an emphasis on health care maintenance and chronic disease management (Smith et al., 2014). Several SRCs have offered mental health screening and care as

Jessica Spellun and Jess Zonana are co-principal investigators.

Constance Zhou

coz2002@med.cornell.edu

1 Weill-Cornell/Rockefeller/Sloan-Kettering Tri-Institutional MD-PhD Program, 1300 York Ave, Room C-103, New York, NY 10065, USA

2 Weill Cornell Medicine, 1300 York Ave, Room C-118, New York, NY 10065, USA

3 Weill Cornell Medicine, 525 East 68th Street, Box 140, New York, NY 10065, USA increasing needs for these services are recognized (Batra et al., 2009; Doyle et al., 2012; Fischbein et al., 2020; Khonsari et al., 2019; Knoll et al., 2021; Liberman et al., 2011; Mann et al., 2019; Mishan \& Dragatsi, 2017; Schweitzer \& Rice, 2012; Soltani et al., 2015; Stuard et al., 2019).

There is significant variation in how SRCs dedicate resources to the psychiatric needs of their patient populations. One approach is to implement psychiatric symptom screening protocols for established or new medical patients, which are followed by referral to community mental health resources (Stuard et al., 2019). Another is to implement mental health screening followed by a combination of medication management and specialist referrals to comprehensive psychiatric care (Liberman et al., 2011). However, many patients have difficulties connecting to affordable and timely care. Patients from racial, ethnic, and sexual and gender minorities experience barriers to accessing care in addition to stress related to prejudice and discrimination, which are risk factors for mental health disorders (Bostwick et al., 2014; Lee et al., 2016; Mays et al., 2007; Williams et al., 2003). To expand psychiatric services in primary care settings, some clinics have developed a model in which medication management occurs under the direct guidance of a consulting psychiatrist (Batra et al., 2009; Mishan \& Dragatsi, 2017). SRCs have also begun to incorporate dedicated 
mental health treatment teams, allowing psychiatrists to work directly with medical students to provide medication management services with supportive interventions (Doyle et al., 2012; Khonsari et al., 2019; Knoll et al., 2021; Mann et al., 2019; Schweitzer \& Rice, 2012; Soltani et al., 2015).

In an effort to provide interdisciplinary psychiatric care, a small number of mental health SRCs include counseling from local non-profits and social work services (Batra et al., 2009; Khonsari et al., 2019; Knoll et al., 2021; Liberman et al., 2011; Schweitzer \& Rice, 2012; Soltani et al., 2015). These comprehensive treatment programs provide patients the benefit of holistic care while offering opportunities for trainees from social work, as well as medical and pharmacy schools to learn collaboratively (Robinson et al., 2004). Interdisciplinary mental health programs have expanded the traditional SRC model but remain the minority among student-led mental health initiatives.

In this paper, we present the Weill Cornell Medicine Wellness Qlinic (Wellness Qlinic) as an innovative model that incorporates interdisciplinary trainees with direct supervisory support to provide comprehensive, culturally responsive, and evidence-based services within the SRC setting. The Wellness Qlinic aims to address the mental health needs of lesbian, gay, bisexual, transgender and queer (LGBTQ+)-identifying patients in New York City. LGBTQ +-identifying patients are at higher risk for suicide, anxiety, depression, and substance use, and are also more likely to encounter barriers accessing adequate psychiatric care (Cochran et al., 2003; Friedman et al., 2014; Kerridge et al., 2017; King et al., 2008; Moagi et al., 2021).

The purpose of the paper is to present a student-run mental health program incorporating interdisciplinary students and trainees to provide multiple modalities of psychiatric treatment, including medication management, time-limited individual psychotherapy, group therapy, and referral services to longer-term treatment when appropriate. We are unaware of any other SRCs dedicated to providing mental health care to LGBTQ +-identifying patients free of charge and regardless of insurance status. Here, we describe the Wellness Qlinic's clinical model, including service delivery design and volunteer involvement, and demonstrate using chart-review data that the model results in high patient retention rates and positive clinical outcomes.

\section{Methods}

The clinic's patient population consists of adults (18 or older) who identify as LGBTQ + and live in New York City. Uninsured and underinsured patients are prioritized. Since opening in March 2019, the Wellness Qlinic has longitudinally served over 50 patients, and has provided a range of clinical services including medication management, short-term individual psychotherapy, and DBT-based group therapy.

This initiative was founded in 2017 by a team of medical students, psychiatry residents, and attending psychiatrists at Weill Cornell Medicine and New York-Presbyterian Hospital, with the support of many other faculty members, students, and staff. In addition to clinical care, the mission of the Wellness Qlinic also includes creating educational and research opportunities for interdisciplinary trainees of all levels to improve LGBTQ + mental health competency. We designed and evolved our organizational structure to reflect our mission, promote efficiency, and establish an infrastructure for sustainable growth (Fig. 1). Three branches were established: Academic Team, Operations Team, and Clinical Team, each overseen by senior student executive leadership and supervised by faculty (Fig. 1). All teams meet as a group with an attending faculty director twice each month to facilitate communication and collaboration. Each team also functions autonomously with separate meetings and specific deliverables. Faculty oversight and supervision is provided to students in executive leadership positions through formal meetings and informal communication channels and is available as needed to all other students. Medical students serve as "board members" in a variety of roles related to education, research, finance, communications/outreach, events, clinical operations, and patient services. To date, over 50 students have volunteered as board members and/or clinical volunteers.

The Academic team designed and executed a mandatory curriculum for volunteers that covers introductory principles and topics in LGBTQ +-affirming treatment and acclimates students to the psychiatric assessment. The Academic team also oversees research projects and facilitates public health and treatment-oriented studies at the medical school and community level. The Operations team manages finances, active grants and future applications, outreach initiatives, fundraising, and event planning. Finally, the Clinical Care team created and implemented our clinical model and coordinates the ongoing operations of volunteer-based patient care delivery, working closely with volunteering attending physicians, psychiatry residents/fellows, psychology interns, and social workers.

All volunteers, regardless of training level, received instruction in LGBTQ +-affirming care. Topics included sensitive and culturally responsive language use, a developmental perspective on sexual orientation and gender identity through the lifespan, identity-affirming transgender care, and an overview of disparities contributing to increased mental illness burden in the LGBTQ + population. Additional clinical didactic instruction on psychiatric assessment and psychotherapy was provided to medical students and trainees according to level of training. Medical students who led group therapy also participated in a separate DBT-based 


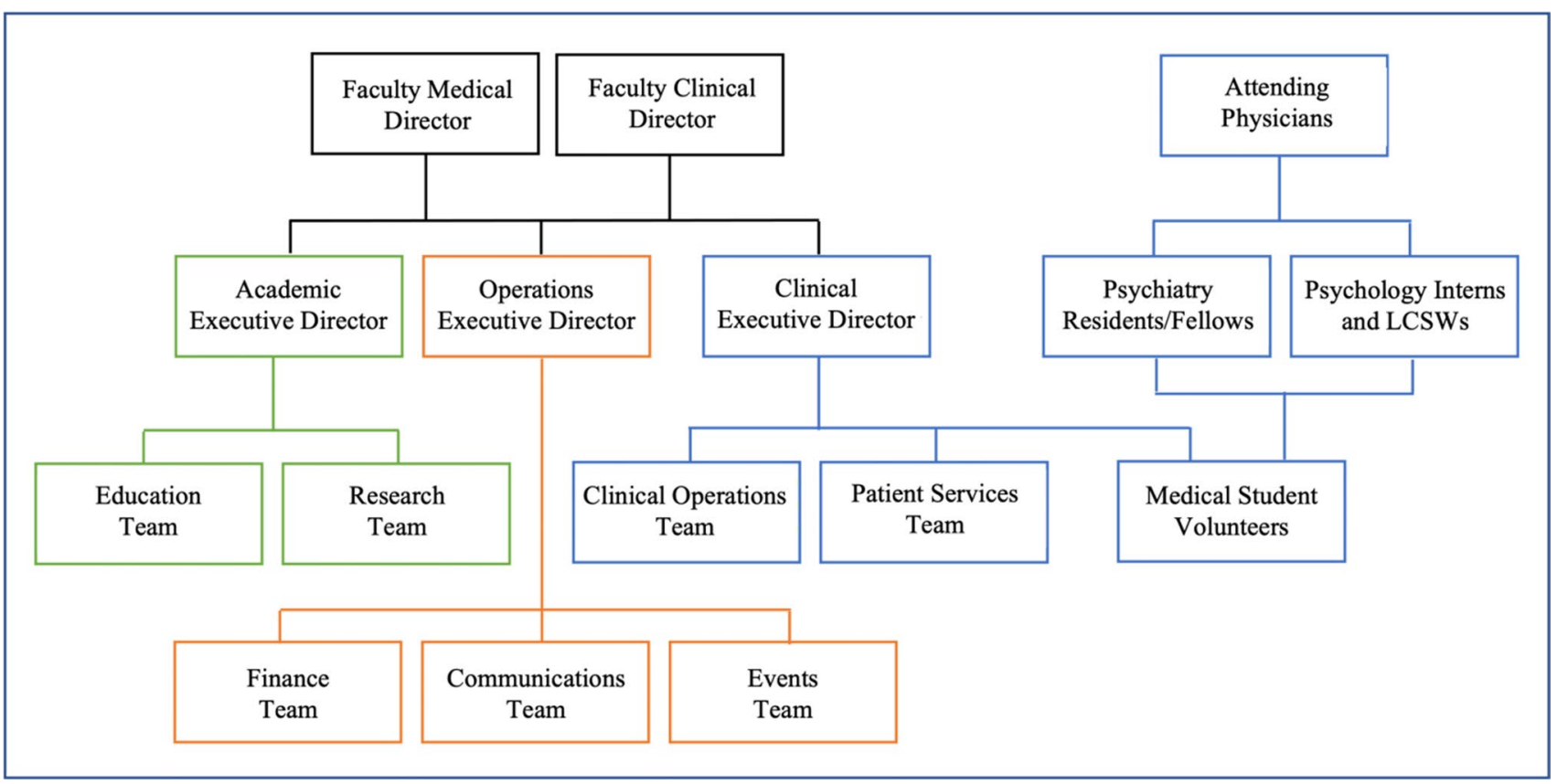

Fig. 1 The Wellness Qlinic organizational structure. The academic team (green) includes the education team and the research team. The operations team (orange) includes the finance, communications, and

skills training in a nested system, where past group leaders trained future group leaders under the supervision of a certified DBT therapist.

A common consideration for all SRCs is navigating risk management and incorporating adequate safeguards within their clinical models. The Wellness Qlinic's supervisory model is in the tradition of SRCs, incorporating both direct and indirect supervision based on level of training, with risk management built into all levels of the clinical workflow. Medical students are trained to participate in the initial triage of new patients, reviewing pertinent details of the patient's history and chief complaint, with an emphasis on suicide and safety assessment. Cases are then reviewed with an attending psychiatrist and patients are offered intake appointments if the services offered by the Wellness Qlinic are suited to their symptom acuity and treatment goals. Patients who are categorized as moderate or high risk by the Columbia Suicide Severity Rating Scale (CSSRS), patients whose illness severity warrants treatment more than one night a week, patients seeking long-term psychotherapy, or patients in need of more comprehensive substance-use treatment, are offered referrals and connected to other treatment in the community.

New patient evaluations and medication management visits are staffed by teams of medical students and psychiatry residents under direct supervision of volunteer attending psychiatrists, who provide guidance and feedback on treatment plans and remain available in case of safety concerns. events teams. The clinical team (blue) includes clinical operations and patient services team. All clinical activities are supervised by attending psychiatrists (Color figure online)

Individual psychotherapy appointments are primarily staffed by psychiatry residents, child and adolescent psychiatry fellows, LCSWs, and psychology interns in a one-on-one setting, with individual therapy supervision by volunteer attending physicians and psychologists. Group therapy sessions are led by a pair of first- or second-year medical students under the direct supervision of a LCSW or a psychiatry resident. All patient appointments are debriefed with a volunteer attending psychiatrist in a mandatory office-hours setting in which students and residents discuss the clinical plan, identify potential safety concerns, and ask questions about the case. Our clinical care model prioritizes both quality of patient care and clinician education, centers medical students as providers, and promotes LGBTQ+ health care literacy for all volunteers.

To assess the efficacy of our model, we performed a chartreview study to evaluate patient engagement and treatment outcomes. The study protocol was reviewed and approved for exemption by the IRB committee of Weill Cornell Medicine. All patient data were deidentified prior to analysis and confidentiality was strictly maintained. Inclusion criteria in the study consisted of patients who requested an appointment, completed a phone screen, and participated in an initial appointment for psychiatric evaluation. The patient data collected consisted of demographic information, services accessed, retention/drop-out rates, and clinical questionnaire scores (PHQ-9 and GAD-7 scores at initial intake and approximately 12 weeks after beginning care). Volunteer 
data was collected, including number of clinical volunteers, their roles in the clinic, and their levels of training.

\section{Results}

Approximately 100 providers and trainees have been involved in the Wellness Qlinic, including: 50 medical students, 20 psychiatry residents, 6 child and adolescent psychiatry fellows, 12 attending psychiatrists, 4 psychology interns and psychology fellows, 2 social workers, 1 graduate student and 2 pre-medical students. Medical students served as both clinical volunteers and board members.

Resident time is a highly valued resource for departments. Adjustments to post-graduate year 2 (PGY2) psychiatry resident responsibilities due to COVID-19 allowed for the opportunity to create a newly developed mandatory rotation with the Wellness Qlinic where they also engage in outpatient psychotherapy and medication management earlier in training. All residents participate in short-term therapy (10-12 sessions) with a patient and are expected to complete four new evaluation or medication management visits as well. At the start of their rotation, all PGY2s receive didactic instruction on introductory principles in supportive psychotherapy. Each resident is paired with an attending psychiatrist or psychologist for weekly individual psychotherapy supervision for the duration of the rotation.

Senior clinical trainees also include PGY4 psychiatry residents, Child/Adolescent psychiatry fellows, psychology interns and psychology fellows participating in an elective. Psychology trainees receive routine individual psychotherapy supervision by attending psychologists. For evaluation and medication management visits, residents and fellows are paired with medical students to provide teaching and mentorship. On date of service, all patient treatment plans including safety planning are reviewed in mandatory office hours format with the precepting volunteer attending. The open office hours format also allows for students to learn about and participate in oral patient presentations and treatment decisions.

Over 28 months of clinical operation, the Wellness Qlinic received approximately 160 appointment requests. A total of 49 patients fulfilled inclusion criteria for this study, all of whom self-identified as LGBTQ + and completed an initial evaluation appointment following our screening process. Of the 49 patients assessed, 6 patients were identified during initial evaluation as needing alternative services and were connected to appropriate care. After completing an evaluation, 38 patients enrolled in treatment and were provided with medication management, individual psychotherapy, and/or group therapy (Fig. 2a). 34 patients (90\%) received short-term psychotherapy services, 26 patients (68\%) received medication management services, and 12 patients (31\%) received group therapy services (Fig. 2b). 26 patients $(68 \%)$ utilized more than one modality of care. For patients receiving individual psychotherapy, $41 \%$ also received psychopharmacology services, $6 \%$ also received group therapy, and $24 \%$ received all three services (Fig. 2a).
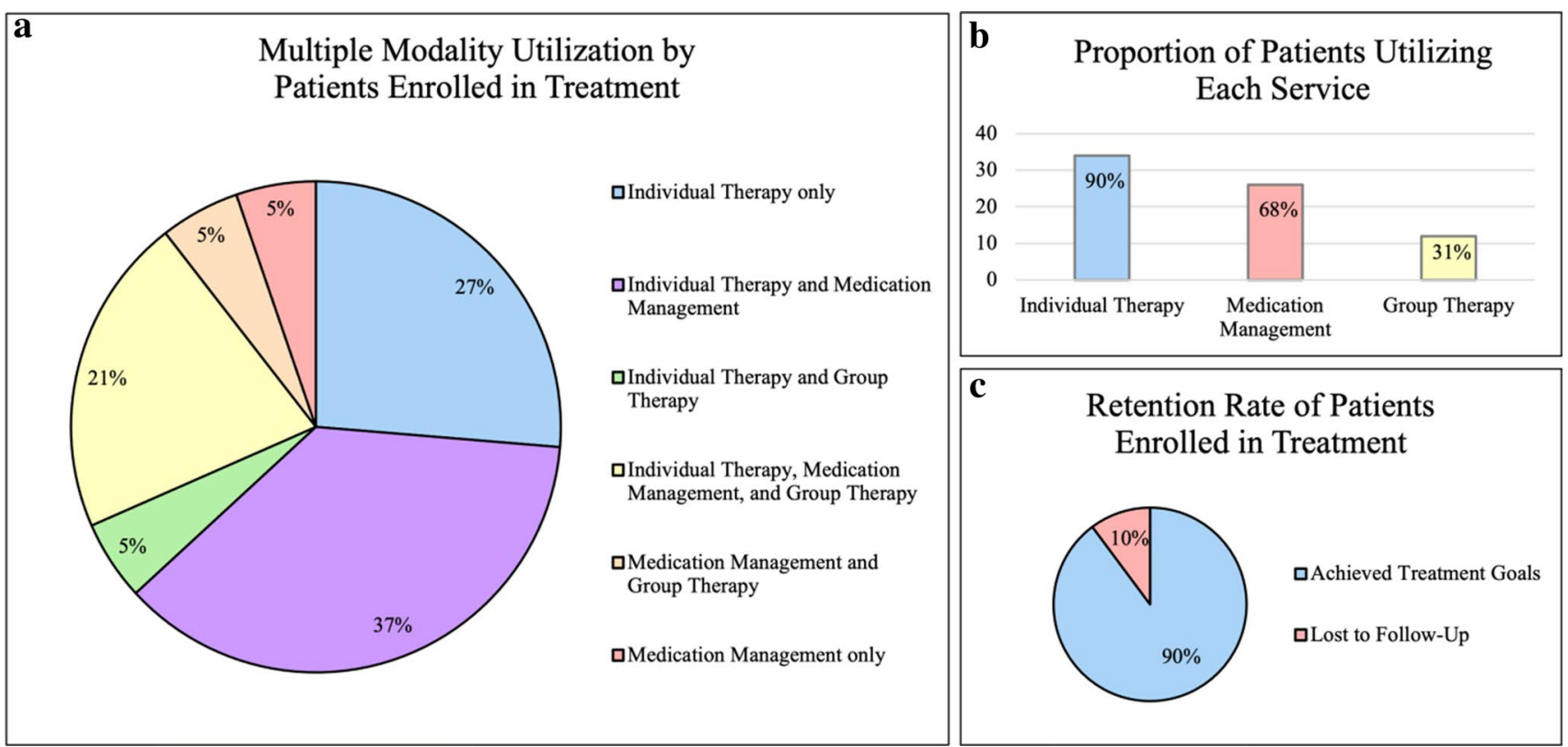

Fig. 2 Service distribution and patient retention. a Proportion of patients receiving services at the Wellness Qlinic who received individual psychotherapy only, medication management only, as well as a combination of $2+$ modalities of care $(n=38)$. $\mathbf{b}$ Number of patients receiving individual psychotherapy, medication management, and group therapy. c Proportion of patients completing treatment 
Patients were made aware that we provide short term psychotherapy averaging 12 weeks in duration, with providers tailoring their sessions to the individual needs and goals of the patients. Patients interested in pursuing additional longterm psychotherapy or other longitudinal behavioral health services were referred to community mental health resources as well. Notably, only 5 patients (10\%) were lost to follow-up at 12 weeks, lower than previous assessments of outpatient psychiatry drop-out rates, which range from 15 to $30 \%$ (Olfson et al., 2009) (Fig. 2c).

The COVID-19 pandemic forced a transition to telehealth appointments. For the Wellness Qlinic, conversion to telehealth expanded our volunteer force, allowing for increased resident and attending participation. Similarly, virtual care reduced travel time and proved convenient for many of our patients who live in the outer boroughs of New York City and worked non-traditional hours. As mental health care needs rose, particularly for sexual and gender minorities, our increased volunteer staff allowed us to provide more patient evaluations and psychiatric services (Druss, 2020; Pfefferbaum \& North, 2020; Salerno et al., 2020; Whaibeh et al., 2020). An important factor contributing to the flux of new patients and visit numbers is fluctuating volunteer availability with transitions in the academic calendar.

Wellness Qlinic patients carry a broad range of diagnoses grouped into categories: depressive disorders, anxiety disorders, bipolar disorders, substance use disorders, neurodevelopmental/attention disorders, eating disorders, personality disorders, and trauma and stress-related disorders. Of these diagnoses, the most prevalent were depressive disorders (27 patients) and anxiety disorders (26 patients). Regardless of diagnosis, all patients completed the PHQ-9 and GAD-7 questionnaires at the beginning of their appointments, allowing for longitudinal symptom tracking. Questionnaires were administered in writing or verbally, at in-person and virtual appointments respectively. Answers were collected and recorded by clinical volunteers.

On average, patients experienced a 5.1-point reduction in their PHQ-9 scores (median: -4 points) and a 6.7-point reduction in their GAD-7 scores (median: -6 points) after 12-weeks of treatment (Figs. 3a, b). These reductions in scores were significant $(\mathrm{p}<0.05)$ by paired two-sample $\mathrm{t}$-tests ( $\mathrm{p}$-values $=1.2 \mathrm{e} 5$ and 0.008 for total PHQ-9 score and total GAD-7 score, respectively). Additionally, patients
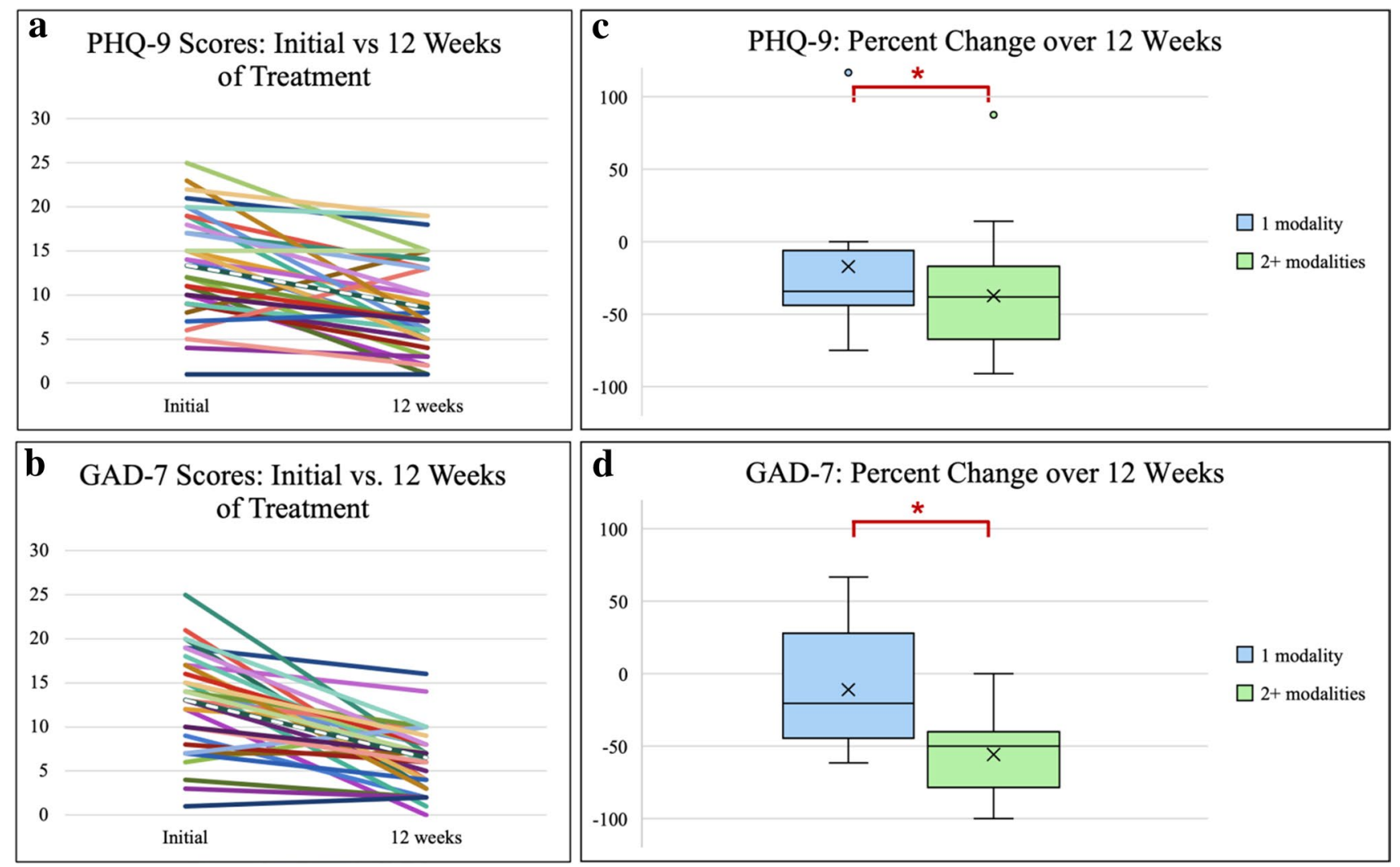

Fig. 3 Reduction in PHQ-9 and GAD-7 Scores Following 12 Weeks of Treatment. a, b Patients on average experienced a 5.1-point reduction in their PHQ-9 and a 6.7-point reduction in their GAD-7 scores after 12 weeks of treatment. Dotted line indicates mean trend line. c, d Patients participating in more than one modality of care experienced a greater percent reduction in PHQ-9 and GAD-7 scores compared to patients enrolled in one modality alone (statistical significance determined by paired-two sample t-test, $\mathrm{p}<0.05$ ) 
experienced a statistically significant ( $\mathrm{p}$-value $=5.4 \mathrm{e} 7$ by paired two-sample t-test) average reduction of 0.5 points for question 7 of the PHQ-9 which assesses frequency of suicidal ideation over the past 2 weeks. This finding is especially important given that LGBTQ + people experience disproportionately high rates of suicidality (Williams et al., 2021). Patients receiving two or more modalities of care experienced on average a 37\% reduction in PHQ-9 scores and a 56\% reduction in GAD-7 scores after 12 weeks, compared to a $17 \%$ reduction in PHQ-9 scores and $11 \%$ reduction in GAD-7 scores in patients who received only one modality of care (Fig. 3c, d). While the Wellness Qlinic is a relatively new initiative and our sample size is too small to draw conclusions generalizable to the population, this preliminary data suggests that integration of multiple modalities of care into the SRC model may contribute to clinically significant improvement for our patients and can be considered in planning models of mental health care delivery (BauerStaeb et al., 2021).

\section{Discussion}

Our model and data demonstrate the feasibility of developing and running a student-run mental health clinic that offers comprehensive mental health services, including individual psychotherapy, with high rates of patient retention, clinical improvement of patient symptoms, and an absence of negative patient outcomes. The Wellness Qlinic is an innovative adaptation of the SRC to specifically address mental health disparities in the LGBTQ + community by providing patient-centered and identity-affirming care. We provide a comparatively broad range of services, at and above the standard of care, while maintaining high retention rates. Our training model expands medical education and psychiatric training in LGBTQ + -culturally responsive care, a topic inadequately addressed by current medical school curricula (Obedin-Maliver et al., 2011).

The ability to provide psychotherapy was a priority in establishing this standalone student-run mental health clinic. As most SRCs are staffed by early medical students, it is difficult to train students as sole providers of psychotherapy. By leveraging the education goals of our institution's psychiatry training programs, we were able to create a rotation for all PGY2 psychiatry residents to gain early exposure to outpatient skills while retaining an emphasis on medical student participation and education. Similarly, we incorporated child and adolescent psychiatry fellows, as well as psychology interns and fellows, with the creation of an elective rotation. Through this adaptation, we successfully offered individual psychotherapy services to $65 \%$ of our patients. We also trained medical students to lead a DBT-based Coping Skills group which has been a valuable learning opportunity and expanded our psychotherapy service offerings. Our data support our initial prioritization of comprehensive treatment options which can be individually tailored.

In assessing our services, we found that patients experienced statistically significant reductions in PHQ-9 and GAD-7 scores 12 weeks into treatment, as determined by paired two-sample t-test. We demonstrated that $68 \%$ of our patients enroll in more than one modality of care, and that those who participate in multiple modalities experience, on average, a greater reduction in PHQ and GAD scores. Interestingly, $17 \%$ of patients who requested care with the Wellness Qlinic reported that this was their first time seeking psychiatric services, in many cases citing concerns surrounding providers' familiarity with LGBTQ + patients and experiences. This finding suggests that we are engaging patients who are new to mental health treatment in a community experiencing cultural mistrust of the medical system (Owens et al., 2007; Shipherd et al., 2010). Additionally, 33\% of patients requesting care from the Wellness Qlinic are uninsured, whereas the average uninsured rate in New York City in 2020 was approximately $11 \%$, suggesting that we serve a community with higher financial and insurance-related barriers to accessing care ("US Census Bureau", 2020).

There are several limitations of our chart-review study including the small number of patients represented and the absence of a control group in any data analysis conducted to measure clinical outcomes. The absence of a non-treated control group narrows the scope of conclusions we can draw from patient PHQ-9 and GAD-7 data and prevents us from determining to what degree clinical improvement is attributable solely to Wellness Qlinic mental health services. However, data showing efficacy of our clinical model are bolstered by our high retention rates, which suggest patient satisfaction towards the quality of care received.

A common criticism of SRCs is that they provide educational opportunities to medical students at the risk of compromising quality of care to uninsured and already marginalized patient populations. Our data demonstrates that comprehensive mental health treatment via an expansion of the SRC model is possible, and allows for statistically significant, positive outcomes as well as patient satisfaction. Building infrastructure for provider education and supervision at multiple levels has enabled the Wellness Qlinic to provide quality mental health care. Furthermore, our $90 \%$ patient retention rate exceeds previously reported averages for outpatient psychiatric care (Olfson et al., 2009). Our findings also suggest that our model's ability to provide multiple treatment modalities, including psychotherapy, contributed to our positive clinical outcomes. Additionally, the educational mission of the Wellness Qlinic emphasizes experiential learning in LGBTQ + care that can translate to other clinical specialties. Clinical 
experience is key to developing culturally responsive care and yields positive clinical outcomes.

A limitation of this and other SRCs is the reliance on volunteers who serve as both clinical providers and the administrative backbone of the clinic. One bottleneck to increasing patient capacity and the breadth of services is volunteer workforce size, especially faculty, who volunteer unpaid hours while maintaining other academic and clinical obligations. The sustainability of a free service model like ours may be contingent on salaried roles for faculty overseers and an organizational structure to support the pursuit of grant opportunities, institutional funding sources, and ongoing philanthropic fundraising. We have found telehealth to be an opportunity for the SRC model to bolster volunteer staffing.

We hope that as the Wellness Qlinic expands, we can continue to address existing disparities in mental health care quality and access, while providing much-needed LGBTQ + education and clinical experience to medical trainees. Other programs can consider this model to offer psychotherapy along with other psychiatric services to underserved communities utilizing the standalone mental health SRC model.

Acknowledgements The authors would like to thank Dr. Marlin Mattson, Dr. Gwen Zornberg, and Dr. Curtis Cole for their advice and support, as well as Dr. Kenny Chen, Alisha Dua, Dr. Jason Harris, Dr. Daniel Huang, Camila Villasante, Daniel Alfonso, Kirsten Bredvik, Andrew Duong, Alex Koo, Jez Marston, Anthony Palillo, Anson Wang, and Bobby Wozniak, and all who have volunteered with and supported the Wellness Qlinic.

Author contributions All authors contributed to the study conception and design. Material preparation, data collection and analysis were performed by $\mathrm{CZ}$ and SS. The first draft of the manuscript was written by $\mathrm{CZ}$ and $\mathrm{KF}$, and all authors commented on previous versions of the manuscript. All authors read and approved the final manuscript.

Funding The Weill Cornell Medicine Wellness Qlinic has received financial support from the APA Helping Hands Grant and the AAMC NEXT Award. Constance Zhou, Sarah Szwed and Matthew Wickersham were supported by a Medical Scientist Training Program grant from the National Institute of General Medical Sciences of the National Institutes of Health under award number T32GM007739 to the Weill Cornell/Rockefeller/Sloan Kettering Tri-Institutional MDPhD Program.

\section{Declarations}

Conflict of interest The authors declare that they have no conflict of interest and certify responsibility for the manuscript.

Ethical approval This research study was conducted retrospectively from data obtained for clinical purposes. We consulted extensively with the IRB of Weill Cornell Medicine, who determined that our study met criteria for IRB exempt status. An IRB official waiver of ethical approval was granted from the IRB of Weill Cornell Medicine.

\section{References}

Batra, P., Chertok, J. S., Fisher, C. E., Manseau, M. W., Manuelli, V. N., \& Spears, J. (2009). The Columbia-Harlem homeless medical partnership: A new model for learning in the service of those in medical need. Journal of Urban Health, 86(5), 781-790. https:// doi.org/10.1007/s11524-009-9386-Z

Bauer-Staeb, C., Kounali, D.-Z., Welton, N. J., Griffith, E., Wiles, N. J., Lewis, G., et al. (2021). Effective dose 50 method as the minimal clinically important difference: Evidence from depression trials. Journal of Clinical Epidemiology, 137, 200-208. https://doi.org/ 10.1016/j.jclinepi.2021.04.002

Bostwick, W. B., Meyer, I., Aranda, F., Russell, S., Hughes, T., Birkett, M., \& Mustanski, B. (2014). Mental health and suicidality among racially/ethnically diverse sexual minority youths. American Journal of Public Health, 104(6), 1129-1136. https://doi.org/10.2105/ AJPH.2013.301749

Cochran, S. D., Mays, V. M., \& Sullivan, J. G. (2003). Prevalence of mental disorders, psychological distress, and mental health services use among lesbian, gay, and bisexual adults in the United States. Journal of Consulting and Clinical Psychology, 71(1), 53-61. https://doi.org/10.1037//0022-006x.71.1.53

Doyle, M. A., Caplan, J. P., \& Marcil, W. (2012). A student-run psychiatry clinic and its use for a medical-student training experience. Academic Psychiatry, 36(3), 237-239. https://doi.org/10. 1176/appi.ap.09060100

Druss, B. G. (2020). Addressing the COVID-19 pandemic in populations with serious mental illness. JAMA Psychiatry. https://doi. org/10.1001/jamapsychiatry.2020.0894

Fischbein, R., Gardner-Buckshaw, S., Loucek, A., Ravichandran, S., Eicher, M., \& Boltri, J. M. (2020). Pandemic productivity: Student-run free clinic integrates behavioral health in the wake of COVID-19. Academic Psychiatry. https://doi.org/10.1007/ s40596-020-01368-w

Friedman, M. R., Dodge, B., Schick, V., Herbenick, D., Hubach, R., Bowling, J., et al. (2014). From bias to bisexaul health disparities: Attitudes toward bisexaual men and women in the United States. LGBT Health, 1(4), 309-318. https://doi.org/10.1089/lgbt.2014. 0005

Kerridge, B. T., Pickering, R. P., Saha, T. D., Ruan, W. J., Chou, S. P., Zhang, H., et al. (2017). Prevalence, sociodemographic correlates and DSM-5 substance use disorders and other psychiatric disorders among sexual minorities in the United States. Drug and Alcohol Dependence, 170, 82-92. https://doi.org/10.1016/j.druga lcdep.2016.10.038

Khonsari, N., Davis, K., \& Wolf, D. (2019). Implementing a psychiatry clinic in a student-run setting: A medical student perspective. Academic Psychiatry, 43(3), 340-343. https://doi.org/10.1007/ s40596-019-01019-9

King, M., Semlyen, J., Tai, S. S., Killaspy, H., Osborn, D., Popelyuk, D., \& Nazareth, I. (2008). A systematic review of mental disorder, suicide, and deliberate self harm in lesbian, gay and bisexual people. BMC Psychiatry, 8, 70. https://doi.org/10.1186/ 1471-244X-8-70

Knoll, O., Chakravarthy, R., Cockroft, J. D., Baddour, N., Jordan, S., Weaver, E., et al. (2021). Addressing patients' mental health needs at a student-run free clinic. Community Mental Health Journal, 57(1), 196-202. https://doi.org/10.1007/s10597-020-00634-3

Lee, J. H., Gamarel, K. E., Bryant, K. J., Zaller, N. D., \& Operario, D. (2016). Discrimination, mental health, and substance use disorders among sexual minority populations. LGBT Health, 3(4), 258-265. https://doi.org/10.1089/lgbt.2015.0135

Liberman, K. M., Meah, Y. S., Chow, A., Tornheim, J., Rolon, O., \& Thomas, D. C. (2011). Quality of mental health care at a studentrun clinic: Care for the uninsured exceeds that of publicly and 
privately insured populations. Journal of Community Health, 36(5), 733-740. https://doi.org/10.1007/s10900-011-9367-5

Mann, C. L., Rifkin, R. A., Nabel, E. M., Thomas, D. C., Meah, Y. S., \& Katz, C. L. (2019). Exploring antidepressant adherence at a student-run free mental health clinic. Community Mental Health Journal, 55(1), 57-62. https://doi.org/10.1007/ s10597-018-0301-5

Mays, V. M., Cochran, S. D., \& Barnes, N. W. (2007). Race, race-based discrimination, and health outcomes among African Americans. Annual Review of Psychology, 58, 201-225. https://doi.org/10. 1146/annurev.psych.57.102904.190212

Mishan, L. I., \& Dragatsi, D. (2017). Student-run clinics: A novel approach to integrated care, teaching and recruitment. Community Mental Health Journal, 53(4), 460-463. https://doi.org/10.1007/ s10597-017-0081-3

Moagi, M. M., van Der Wath, A. E., Jiyane, P. M., \& Rikhotso, R. S. (2021). Mental health challenges of lesbian, gay, bisexual and transgender people: An integrated literature review. Health $S A$ = SA Gesondheid, 26, 1487. https://doi.org/10.4102/hsag.v26i0. 1487

Obedin-Maliver, J., Goldsmith, E. S., Stewart, L., White, W., Tran, E., Brenman, S., et al. (2011). Lesbian, Gay, bisexual, and transgender-related content in undergraduate medical education. JAMA, 306(9), 971-977. https://doi.org/10.1001/jama.2011.1255

Olfson, M., Mojtabai, R., Sampson, N. A., Hwang, I., Druss, B., Wang, P. S., et al. (2009). Dropout from outpatient mental health care in the United States. Psychiatric Services (washington, D.c.), 60(7), 898-907. https://doi.org/10.1176/appi.ps.60.7.898

Owens, G. P., Riggle, E. D. B., \& Rostosky, S. S. (2007). Mental health services access for sexual minority individuals. Sexuality Research \& Social Policy, 4(3), 92. https://doi.org/10.1525/srsp. 2007.4.3.92

Pfefferbaum, B., \& North, C. S. (2020). Mental health and the Covid19 pandemic. New England Journal of Medicine. https://doi.org/ 10.1056/nejmp2008017

Robinson, W., Barnacle, R., Pretorius, R., \& Paulman, A. (2004). An Interdisciplinary student-run diabetes clinic: Reflections on the collaborative training process. Families, Systems, \& Health, 22, 490-496. https://doi.org/10.1037/1091-7527.22.4.490

Salerno, J. P., Williams, N. D., \& Gattamorta, K. A. (2020). LGBTQ populations: Psychologically vulnerable communities in the COVID-19 pandemic. Psychological Trauma: Theory, Research, Practice, and Policy, 12, 239-242. https://doi.org/10.1037/tra00 00837

Schweitzer, P. J., \& Rice, T. R. (2012). The student-run clinic: A new opportunity for psychiatric education. Academic Psychiatry, 36(3), 233-236. https://doi.org/10.1176/appi.ap.10110163

Shipherd, J. C., Green, K. E., \& Abramovitz, S. (2010). Transgender clients: Identifying and minimizing barriers to mental health treatment. Journal of Gay \& Lesbian Mental Health, 14(2), 94-108. https://doi.org/10.1080/19359701003622875

Shrader, S., Thompson, A., \& Gonsalves, W. (2010). Assessing student attitudes as a result of participating in an interprofessional healthcare elective associated with a student-run free clinic. Journal of Research in Interprofessional Practice and Education, 1(3), 23.

Simpson, S. A., \& Long, J. A. (2007). Medical student-run health clinics: Important contributors to patient care and medical education. Journal of General Internal Medicine, 22(3), 352-356. https://doi. org/10.1007/s11606-006-0073-4

Smith, S., Thomas, R., 3rd., Cruz, M., Griggs, R., Moscato, B., \& Ferrara, A. (2014). Presence and characteristics of student-run free clinics in medical schools. JAMA, 312(22), 2407-2410. https:// doi.org/10.1001/jama.2014.16066

Smith, S. D., Johnson, M. L., Rodriguez, N., Moutier, C., \& Beck, E. (2012). Medical student perceptions of the educational value of a student-run free clinic. Family Medicine, 44(9), 646-649.

Soltani, M., Smith, S., Beck, E., \& Johnson, M. (2015). Universal depression screening, diagnosis, management, and outcomes at a student-run free clinic. Academic Psychiatry, 39(3), 259-266. https://doi.org/10.1007/s40596-014-0257-x

Stuard, W. L., Squiers, K., Suss, A., Schrader, E., Triantafyllou, D., Brenner, A., \& North, C. S. (2019). Development and implementation of psychiatric services in a student-operated clinic. Community Mental Health Journal, 55(4), 553-560. https://doi.org/ 10.1007/s10597-018-0325-x

US Census Bureau. (2020). American Community Survey (ACS). Retrieved October 29, 2021 from https://www.census.gov/progr ams-surveys/acs

Whaibeh, E., Mahmoud, H., \& Vogt, E. L. (2020). Reducing the treatment gap for LGBT mental health needs: The potential of telepsychiatry. The Journal of Behavioral Health Services \& Research, 47(3), 424-431. https://doi.org/10.1007/s11414-019-09677-1

Williams, A. J., Jones, C., Arcelus, J., Townsend, E., Lazaridou, A., \& Michail, M. (2021). A systematic review and meta-analysis of victimisation and mental health prevalence among LGBTQ+ young people with experiences of self-harm and suicide. PLOS ONE, 16(1), e0245268. https://doi.org/10.1371/journal.pone.0245268

Williams, D. R., Neighbors, H. W., \& Jackson, J. S. (2003). Racial/ ethnic discrimination and health: Findings from community studies. American Journal of Public Health, 93(2), 200-208. https:// doi.org/10.2105/ajph.93.2.200

Publisher's Note Springer Nature remains neutral with regard to jurisdictional claims in published maps and institutional affiliations. 